\title{
Simple bone cyst in the calcaneus. About a case
}

\author{
Cristina Gonzalez-martin ${ }^{1 *}$, Pilar Bolsa-Col ${ }^{2}$, Abian Mosquera-Fernandez ${ }^{1}$, Vanesa Balboa-Barreiro ${ }^{3}$ and Daniel Gulias-Soidan ${ }^{4}$ \\ ${ }^{1}$ Clinical Epidemiology Research Group, Health Sciences Department, Faculty of Nursing and Podiatry, Universidade da Coruña (UDC) Campus de Ferrol, España, \\ Spain \\ ${ }^{2}$ Degree in Podology. University of A Coruña, Spain \\ ${ }^{3}$ Research Group of Clinical Epidemiology and Biostatistics, Biomedical Research Institute of A Coruña (INIBIC), University Hospital Complex of A Coruña \\ (CHUAC), Spain \\ ${ }^{4}$ Department of Radiology, University Hospital Complex of A Coruña (CHUAC), Spain
}

\begin{abstract}
Background and Objective: The presence of the simple bony cyst is a rare condition in the foot. Their finding may be due to other alterations or pain in that area. The objective of this work is to know the characteristics of simple bone cyst, its treatment and evolution.

Methods: The case we present is that of a 13-year-old male who comes to the clinic due to a sprain. Due to the tests performed, the presence of a simple bony cyst in the calcaneus is accidentally found. It is surgically intervened, making a filling.

Results: A surgical intervention will be carried out, a filling of the cyst area will be performed, due to the complication a new intervention will be carried out.

Conclusion: Simple bone cyst in the calcaneus can cause the collapse of the calcaneus causing a great deformity and disability in the permanent foot.
\end{abstract}

\section{Introduction}

The most outstanding aspects of the foot skeleton in relation to the tumoral lesions, refer to the particular characteristics of the bones that compose it and the poor compartmentalization of the middle and hindfoot, which are considered extra compartmental spaces.

On the contrary, the forefoot is well compartmentalized in five spaces that correspond to the five radios. The fact that the bones are very superficial, the scarce coverage of the soft tissues around, the intimate relationship of the neurovascular and tendinous structures, the compact organization of the small bones, the fineness of their cortex and the abundant spongy component in some of the they will facilitate extraosseous tumor growth [1].

Most tumors that occur in the foot and ankle are reactive and inflammatory, however, due to their rarity, primary and metastatic neoplasms are a diagnostic concern since they can often go unnoticed [2].

Bone tumors of the ankle and foot account for 1-5\% of all skeletal tumors. In the foot appear less than $2 \%$ of the total and most are benign, showing some of them a particular predisposition for bones or specific regions $[1,3]$.

Most tumors are located in the middle and hindfoot. As for the forefoot, the most frequent would be in the phalanges [1].

As more frequent benign tumor lesions would be found; osteoid osteoma, giant cell tumors, subungual exostosis, simple bone cysts, osteochondroma and chondromas [1].

Regarding malignant tumor lesions, they would stand out; osteosarcodroma, chondrosarcoma, Ewing sarcoma, bone lymphomas, myeloma, and metastasis, mainly, although at the level of the foot are infrequent [1].
The World Health Organization (WHO) published the first classification of bone tumors based mainly on the tissues in which the tumor has developed, and on what tissue it is formed. Pseudotumoral lesions were also classified.

The simple bone cyst (QOS) of the calcaneus is a benign and very rare lesion. It has a pseudotumoral character that can sometimes grow and dilate the cortex, even breaking it. Its diagnosis is usually casual or due to the appearance of pain, sometimes accompanied by swelling. It presents a very low incidence and usually appears at early ages of life, because the growth cartilages are open [4].

The WHO defines it as "a unicameral cavity filled with clear or bloody fluid, covered by a membrane of variable thickness, consisting of loose vascular connective tissue in which scattered osteoclastic giant cells and sometimes areas of recent or ancient hemorrhages or crystals can be observed of cholesterol " [5].

The pain from this injury is due to trabecular and cortical fractures. It usually occurs when the size of the cyst is greater than $1 / 3$ of its mass.

The bony cyst has a very characteristic radiology, radiolucent image with a central osteolysis of well-defined, sclerous and thinned edges, which can expand the cortex, occupying half or $2 / 3$ of the mass of the calcaneus [3].

*Correspondence to: Cristina Gonzalez-martin, Clinical Epidemiology Research Group, Health Sciences Department, Faculty of Nursing and Podiatry, Universidade da Coruña (UDC) Campus de Ferrol, España, Spain, E-mail: cristina.gmartin@udc.es

Key words: bone cyst, calcaneus, treatment

Received: December 11, 2019; Accepted: December 27, 2019; Published: December 31, 2019 
Its definitive diagnosis could be confirmed by CT, MRI and histological study.

The treatment of the QOS, includes the observation and the control of the same, since it can heal spontaneously during the skeletal maturation when producing partial fractures. Corticosteroid infiltrations or the surgical option of choice that consists of a curettage and a bone graft of the cavity can also help. The bone defect or the cavity can be filled with hydroxyapatite or polymethacrylate. Other treatment options could be intralesional percutaneous injection with autologous bone marrow [5].

\section{Case presentation}

A 13-year-old male patient is seen by a primary care provider for pain and inflammation of the right ankle. There is no personal or family history of trauma or other pathological conditions at the level of the foot.

His personal medical history does not indicate any drug allergies, or that he had undergone any surgical interventions before this encounter.

The general clinical examination shows no pathological changes. He presents with swelling in the heel and right hindfoot, with pain on palpation and while standing. During the exploration, we found a morphologically normal foot, with a well-aligned hindfoot. Laboratory tests were within normal parameters.

After the examination, it is determined that his pain and inflammation are compatible with a sprain. An x-ray is requested for a clear diagnosis, and to rule out possible fractures. After visualization of the x-ray, he is diagnosed with a sprain of the lateral ligament of the right ankle.

We incidentally find a calcaneus, a lytic lesion with well-defined edges, which is compatible with a lipoma or simple bone cyst.

A decision is made to conduct magnetic resonance imaging (MRI) of the foot to identify the specific type of injury. The presence of a well-defined lytic lesion is confirmed, approximately $30 \times 22 \times 20 \mathrm{~mm}$ in the anteroinferior part of the calcaneus, with hypointense sequences enhanced on T1, which includes hyperintense sequences with fat suppression.

Findings are compatible with the diagnosis of a simple bone cyst (Figure 1).

It was also decided to perform a magnetic resonance imaging (MRI) of the foot to try to identify the type of injury. The presence of a well-defined lytic lesion was confirmed, approximately $30 \times 22 \times 20$ $\mathrm{mm}$ in the antero-inferior part of the calcaneus, hypo intense in the sequences enhanced in $\mathrm{T} 1$ and hyper intense in the sequences with fat suppression. Findings compatible with the diagnosis of simple bone cyst (Figure 2A,2B).

The size of the cyst can cause the collapse of the subtalar joint, so due to the age of the patient the surgical treatment is indicated to avoid permanent deformities in the architecture of the foot that cause chronic painful sequelae in walking.

\section{Treatment plan}

The factors to take into account at the time of treatment are: the size of the cyst and its activity, the distance it is from the physis and whether it is located in a loading zone or not.

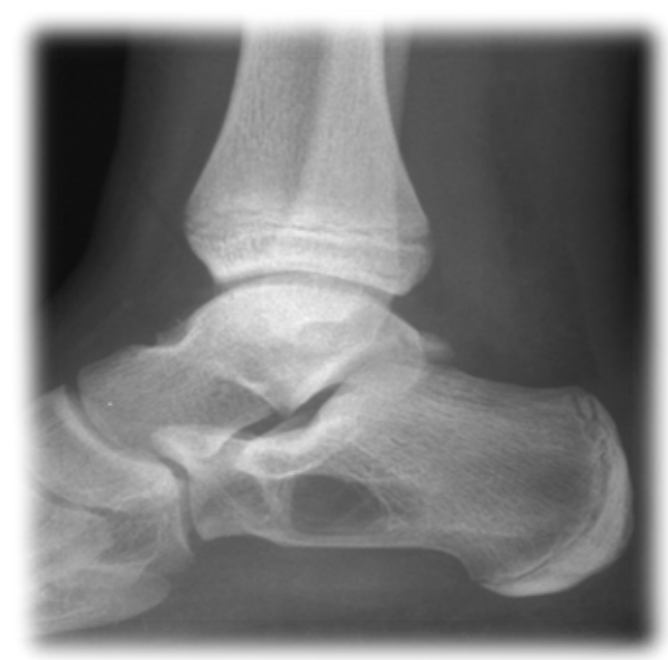

Figure 1. Lytic lesion in calcaneus. Detail of the lateral X-ray of the foot

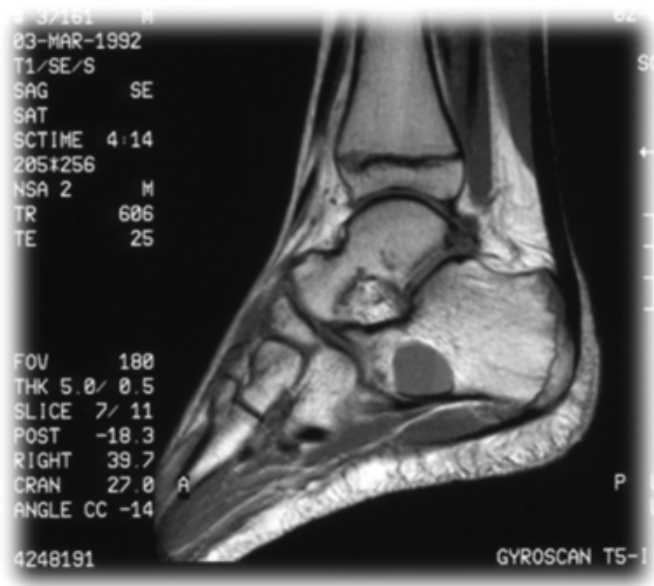

Figure 2A. In T1-weighted sequences, the lesion is well defined and clearly hypointense

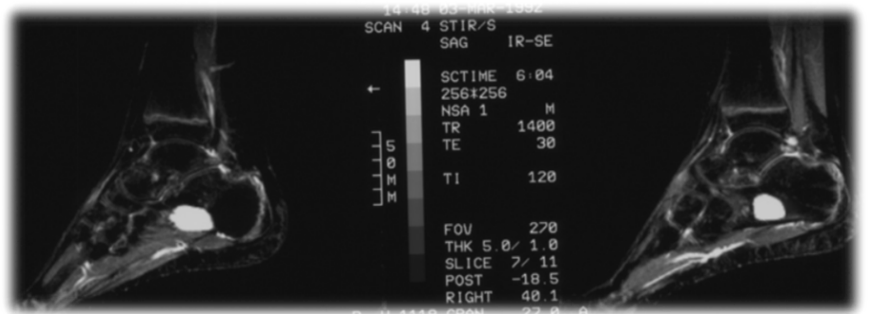

Figure 2B. STIR in which the hyperintensity of the lesion in the calcaneus is appreciated

It is important to always explain that this lesion is benign in nature.

Among the therapeutic possibilities of the bone cyst are the injection of corticosteroids into the cyst, the performance of multiple perforations and drainage of the cystic cavity, the curettage of the membranous wall and the application of bone graft, the subtotal resection with bone graft placement, subtotal resection without bone graft, total ablation (diaphysectomy) and bone graft.

The response to treatment was assessed according to the criteria of Neer, et al. [6]:

A. Complete cure: complete obliteration of the cyst by bone neoformation. 
B. Incomplete healing: bone neoformation that partially fills the cyst, persisting non-progressive radiolucent areas.

C. No response: the cyst does not experience clinical or radiological changes.

D. Recurrence: after complete healing, radiolucent areas reappear that grow, progressively expanding and thinning the cortex.

\section{Intervention and evolution}

Guided radiologically, the cyst is localized with trocars, the cavity is washed with physiological serum, filled with bone plast and intralesional infiltration of corticosteroid type betamethasone.

After the procedure a plaster splint is applied to the patient and it is recommended not to support the foot. The first revision is made 7 days after the intervention. After this first revision, the patient begins to walk with sticks: this process will be carried out for a month. A new revision is scheduled there.

After a month and a half of the intervention, you are recommended to lead a normal life. The next review is not scheduled until 6 months.

In the evolutionary radiological controls, a favorable but incomplete response was observed with a slight sclerosis of the lesion in the simple $\mathrm{X}$-ray (Figure 3 ) and changes in the content of the cyst in the magnetic resonance (Figure 4A,4B).

At the 6-month follow-up, in which MRI had previously been requested, the patient presents pain to the lateral palpation, so a new intervention is performed due to the present symptoms and the data found in the aforementioned tests complementary.

A new intervention is performed, again under sedation and with local infiltration of anesthesia in which the hematic content of the cyst is aspirated and filled with calcium hydroxyapatite and betamethasone.

At 6 months again after the second intervention, the patient no longer presents with painful symptoms, and the complementary tests show a favorable evolution, which means that he is discharged.

In this last revision we checked joint ranges, we performed a muscle assessment, study of the footprint and we found that all parameters are within normal. The patient does not present pain or joint stiffness in any movement.

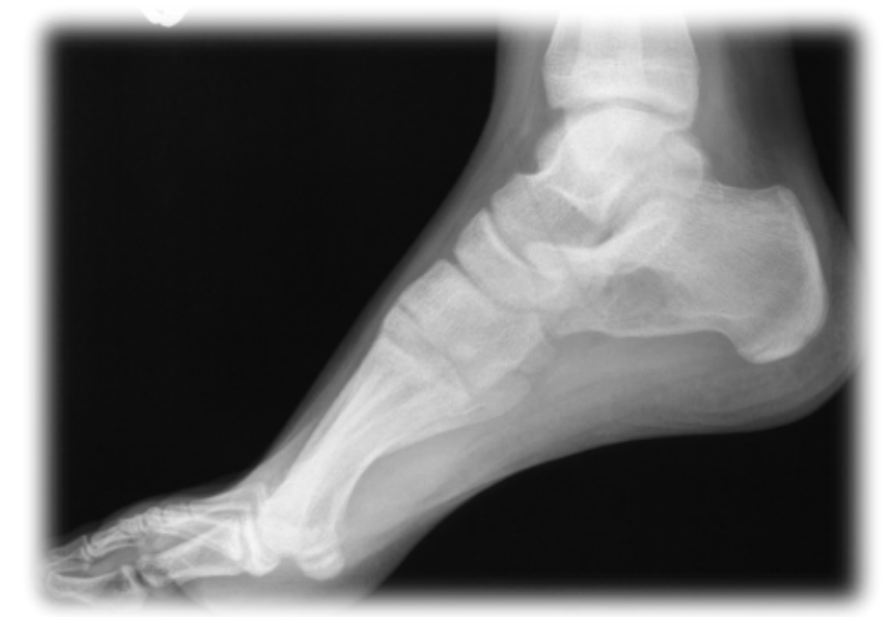

Figure 3. Control X-ray in which a slight sclerosis of the treated lesion is seen, without evidence of extravasation of the filling material or other complications

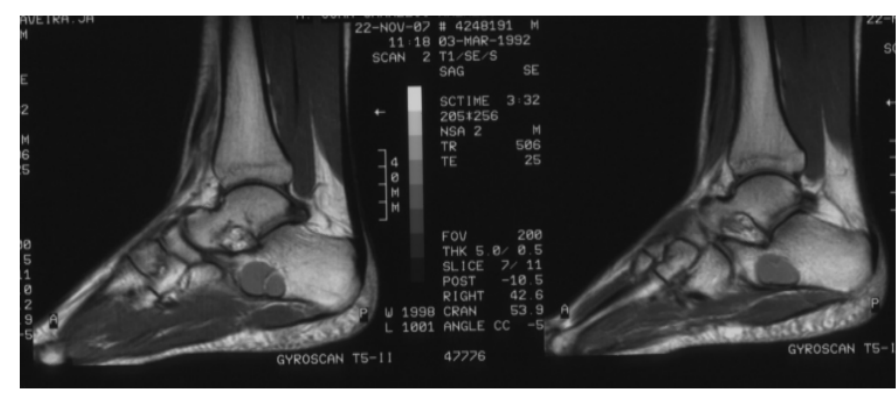

Figure 4A. Sagittal T1 in which the treated lesion is seen, hypointense and already with morphological alterations of its content

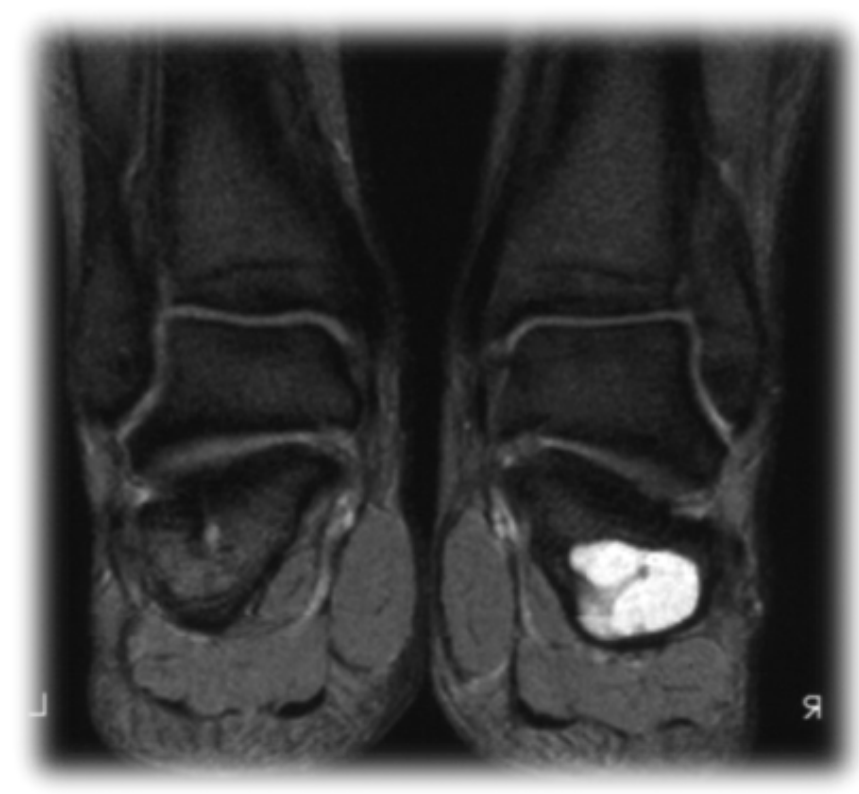

Figure 4B. Coronal STIR. A large hyper signal continues to be observed in the fat suppression sequences

The study of the footprint is quite neutral and stabilized, so that no orthopodological treatment is performed.

On this occasion, the radiological controls at 6 months show a clear sclerosis of the lesion (Figure 5). In the following revisions in consultation the satisfactory clinical evolution of the patient is confirmed by what is discharged.

Before carrying out the assessment, the patient is asked if he or she agreed to obtain a series of data for the preparation of the clinical case. Due to the fact that the patient is a minor, they are authorized by their legal guardians to access the data and to carry out this study. With this consent was obtained so that all the relevant information for the completion of this final degree project could be accessed, explaining that it would not negatively interfere with the care that he was going to receive during the hospitalization, since the assistance I was going to lend it would be the same.

\section{Discussion}

Calcaneal bone cysts have a very low incidence of presentation. Our study coincides with the literature as the form of incidental finding.

The activity of the bony cyst diminishes as the person matures skeletally and as the body growth ends, this way it does not spread 


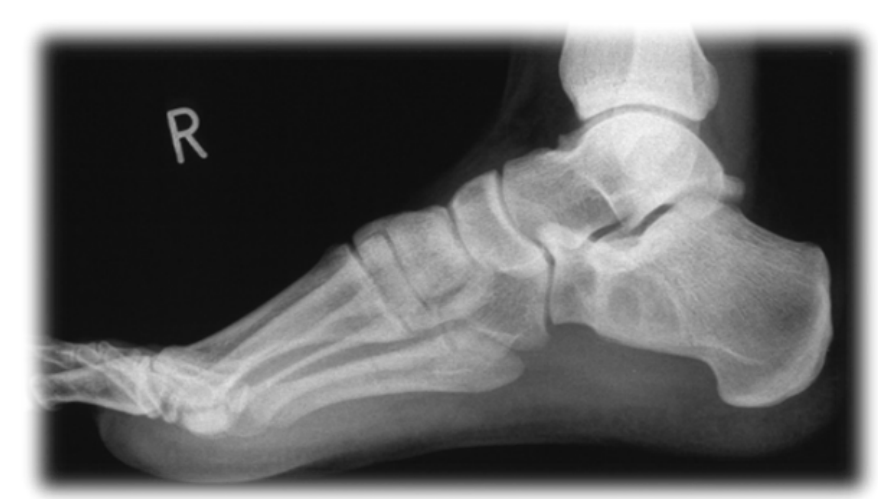

Figure 5. Lateral radiograph of the foot in which the increase in density of the lesion treated is clearly visible

further, spontaneously and slowly reinforcing the cortical cover, not recurring the lesion if has made a correct treatment. Only in exceptional cases are essential bone cysts resolved after a pathological fracture.

The treatment of bone cyst by curettage and application of autologous bone graft is a good alternative for loading bones such as the calcaneus, although according to the consulted literature it has a recurrence that oscillates by $15 \%$.

The differential diagnosis of QOS includes pseudocysts, hemophilic pseudotumors, lipomas, osteomyelitis, aneurysmal bone cysts, nonossifying fibromas, giant cell tumors, solitary eosinophilic granulomas, and malignant hemangioendote-liomas $[7,8]$. The diagnosis is confirmed with computed tomography (CT), MRI and the histological study.

The simple radiographic axial projection does not allow to adequately assess this lesion, therefore lateral projections are made. The radiological follow-up allowed to corroborate the changes of increase of the bone density in the radiographs within the lytic lesion and the thickening of the cortical, as well as the formation of internal trabeculations.

The aim of surgical treatment of QOS in the calcaneus is to reduce the risk of fracture. The healing rate of the QOS in the calcaneus is greater by curettage and graft filling, than with infiltration with corticosteroids, which presents high rates of recurrence [9]. The most effective treatment for calcaneal QOS has proven to be curettage associated with perforations, with the contribution of autologous or heterologous bone graft, associating or not a screw that keeps the cavity open [10].

\section{Conclusion}

With this work we want to highlight that the QOS in the calcaneus is an unusual benign lesion that does not affect the vital prognosis but that the collapse of the calcaneus can cause a great deformity and disability in the permanent foot.

These lesions should be treated surgically, to give a good consistency to the calcaneus, because this is a very important bone in the base of the weight support of the body. Any alteration in this bone can cause important alterations in the individual's gait.

\section{References}

1. Pascua LR, Herráez SS, Hernández ÓF, Díaz AS (2011) Bone tumors of the foot Revista del Pie y Tobillo 25: 6-19.

2. Delgado-Ceillo EA, Rico-Martínez G, Linares-González LM, Estrada-Villaseñor E, Renán-León S, et al. (2007) Epidemiology of bone and soft tissue neoplasms of the foot and ankle. Acta Ortop Mex 21: 144-150.

3. Aguado Hernández HJ, Burón Soto I, Cancho Candela R (2010) Calcaneal simple bone cyst: A potentially disabling unusual "benign" lesion. A Pediatr (Barc) 73: 62-64.

4. Subramanian S, Viswanathan VK (2019) Bone Cyst. http://www.ncbi.nlm.nih.gov/ books/NBK539849/ PubMed PMID: 30969671.

5. Linares-González LM, Rico-Martínez G, Zamudio-Villanueva L (2002) Simple bone cyst of calcaneus and its treatment with autologous bone marrow. Cir Ciruj 70: 109115 .

6. Neer CS, Francis KC, Marcove RC, Terz J, Carborana PN (1966) Treatment of unicameral bone cyst. A follow-up study of 175 cases. J Bone Joint Surg-Am 48: 731745

7. Fernández Portal L (2007) Tumor Pathology Biomechanics, medicine and foot surgery Núñez-Samper M, Llanos Alcázar LF, eds. Barcelona: Elsevier; 315-6.2.

8. Pogoda P, Priemel M, Catalá-Lehnen P, Gebauer M, Rupprecht M, et al. (2004) Simple bone cysts of the calcaneus. Differential diagnosis and therapy. Unfallchirurg 107: 680684.

9. Cuenca Espiérrez J, Herrera Rodríguez A, Gil Albarova J, Martínez Martín AA Bregante Baquero J (2002) The essential bone cyst in childhood. Retrospective study of 15 cases and review of the literature. Cir Pediatr 15: 68-72.

10. Saraph V, Zwick EB, Maizen C, Schneider F, Linhart WE (2004) Treatment of unicameral calcaneal bone cysts in children: review of literature and results using a cannulated screw for continuous decompression of the cyst. J Pediatr Orthop 24: 568573 .

Copyright: (C)2019 Gonzalez-martin C. This is an open-access article distributed under the terms of the Creative Commons Attribution License, which permits unrestricted use, distribution, and reproduction in any medium, provided the original author and source are credited. 